$$
\begin{aligned}
& \text { SANDA-301EC } \\
& \text { SAND- } 96-3015 C
\end{aligned}
$$

\title{
PROPERTIES OF HIGH GAIN GaAs SWITCHES FOR PULSED POWER APPLICATIONS*
}

\author{
Fred J Zutavern, Guillermo M. Loubriel, Harold P. Hjalmarson, Alan Mar, \\ Wesley D. Helgeson, Martin W. O'Malley, Mitchell H. Ruebush RECEIVED \\ Sandia National Laboratories, Albuquerque, NM 87185-1153 \\ (505)845-9128 \\ R. Aaron Falk, OptoMetrix, Inc., Renton, WA 98058 \\ (206) 271-0779

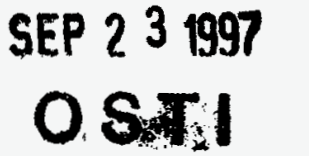

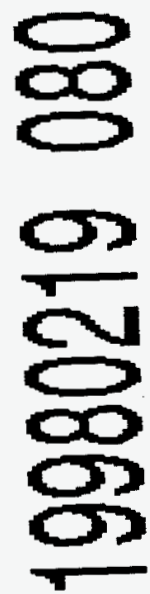

\begin{abstract}
High gain GaAs photoconductive semiconductor switches (PCSS) are being used in a variety of electrical and optical short pulse applications. The highest power application, which we are developing, is a compact, repetitive, short pulse linear induction accelerator. The array of PCSS, which drive the accelerator, will switch $75 \mathrm{kA}$ and $250 \mathrm{kV}$ in $30 \mathrm{~ns}$ long pulses at $50 \mathrm{~Hz}$. The accelerator will produce a $700 \mathrm{kV}, 7 \mathrm{kA}$ electron beam for industrial and military applications. In the low power regime, these switches are being used to switch $400 \mathrm{~A}$ and $5 \mathrm{kV}$ to drive laser diode arrays which produce $100 \mathrm{ps}$ optical pulses. These short optical pulses are for military and commercial applications in optical and electrical range sensing, $3 \mathrm{D}$ laser radar, and high speed imaging. Both types of these applications demand a better understanding of the switch properties to increase switch lifetime, reduce jitter, optimize optical triggering, and improve overall switch performance. These applications and experiments on the fundamental behavior of high gain GaAs switches will be discussed. Open shutter, infra-red images and time-resolved Schlieren images of the current filaments, which form during high gain switching, will be presented. Results from optical triggering experiments to produce multiple, diffuse filaments for high current repetitive switching will be described.
\end{abstract}

\section{Introduction}

The first half of this paper discusses several high speed switching applications that can benefit from some of the advantageous properties of high gain GaAs photoconductive semiconductor switching (PCSS). These applications are: (1) compact, repetitive particle beam accelerators; (2) ground penetrating radar (GPR); (3) optically activated electrical firesets; (4) semiconductor laser diode drivers; (5) electro-optic (Pockels cell) drivers; and (6) high voltage triggers for gas discharge switches. For each application, we briefly describe the purpose, switching goals, benefits from using PCSS, and issues or areas which require improved PCSS operation. From these descriptions, one can see that both benefits and issues depend on the specific application. Additional information on some of these applications can be found in the literature ${ }^{1}$. The second half of the paper reports experiments and calculations that are focused on understanding and improving these switches. Many of the present limitations of high gain GaAs PCSS are being eliminated or reduced by learning more about switch operation, fabricating switches with different types of

Table I. Switching results achieved with high gain GaAs PCSS (not simultaneous).

\begin{tabular}{|c|c|}
\hline maximum power & $60 \mathrm{MW}$ \\
\hline maximum voltage & $155 \mathrm{kV}$ \\
\hline maximum current & $7 \mathrm{kA}$ \\
\hline minimum trigger energy & $2 \mathrm{~nJ}$ \\
\hline maximum trigger gain & $10^{5}$ \\
\hline maximum electric field & $100 \mathrm{kV} / \mathrm{cm}$ \\
\hline minimum rise time & $350 \mathrm{ps}$ \\
\hline minimum r-m-s jitter & $80 \mathrm{ps}$ \\
\hline max. continuous rep. rate & $1 \mathrm{kHz}$ \\
\hline max. burst rep. rate & $5 \mathrm{MHz}$ \\
\hline max. device lifetime & $25,000,000$ \\
\hline
\end{tabular}
* This work was supported by the U.S. Department Of Energy under Contract DE-AC04-94AL85000. Sandia is a multiprogram
laboratory operated by Sandia Corporation, a Lockheed Martin Company, for the United States Department of Energy. 


\section{DISCLAIMER}

This report was prepared as an account of work sponsored by an agency of the United States Government. Neither the United States Government nor any agency thereof, nor any of their employees, make any warranty, express or implied, or assumes any legal liability or responsibility for the accuracy, completeness, or usefulness of any information, apparatus, product, or process disclosed, or represents that its use would not infringe privately owned rights. Reference herein to any specific commercial product, process, or service by trade name, trademark, manufacturer, or otherwise does not necessarily constitute or imply its endorsement, recommendation, or favoring by the United States Government or any agency thereof. The views and opinions of authors expressed herein do not necessarily state or reflect those of the United States Government or any agency thereof. 
electrical contacts, and testing switches in different configurations. A list of switching properties, which we have demonstrated with high gain GaAs PCSS is shown in Table I. A key issue for almost all PCSS applications is device longevity. Most of our research and development in this area is not described in this paper, because it is covered in a companion paper that is devoted to PCSS longevity. ${ }^{2}$

\section{Applications}

Compact, repetitive, short pulse, particle beam accelerators can be used as radiation sources for radiography, materials processing and sterilization of food, medical equipment, and medical waste. They could also be used in biological counter-proliferation to sterilize suspicious packages at the site of discovery. An entry point in these areas is a PCSS driven linear induction accelerator $^{3}$, that we are developing to produce $700 \mathrm{kV}$ and $7 \mathrm{kA}$ in $10-30 \mathrm{~ns}$ long pulses at 50 Hz. The benefits of using high gain PCSS over conventional spark gap based accelerator technology are: size, weight, repetition rate, rise time, and jitter. The issues are switch lifetime, switched current, and stand-off voltage. Continuous operation at $50 \mathrm{~Hz}$ or higher requires switches that will last for $10^{7}$ or more pulses per 40 hour week. Although some of the uses described here would not require continuous operation, the general need for long-lived switches is clear. Switch degradation is exacerbated by the requirements for high currents. Our linear induction accelerator is a modular voltage-adder that requires $56 \mathrm{kA}$ through the switches or 28 $\mathrm{kA}$ and $200 \mathrm{kV}$ into the induction cavities to accelerate $7 \mathrm{kA}$ at $700 \mathrm{kV}$. Our approach to build a PCSS based accelerator with reasonable lifetime $\left(10^{7}\right.$ pulses) has been to limit the current per filament in the PCSS to less than $50 \mathrm{~A}$ by initiating many filaments in each GaAs wafer. We have used line triggering to produce 30 current filaments across a $3 \mathrm{~cm}$ wide GaAs PCSS (Figure 1). We are presently testing a 6 wafer module which will power $1 / 8^{\text {th }}$ of the accelerator. Fiber optics are being used to deliver the optical trigger to the switches and $1 \mathrm{~mm}$ diameter glass rods are being used to focus the light into 100-200 $\mu \mathrm{m}$ wide lines across the active region of the switches. With this design, the full accelerator will be powered with 48 wafers which conduct current in 1440 filaments, or 39 A per filament. More information on line triggering is provided later in this paper under the section on triggering experiments. PCSS lifetime improvements and testing are described in the companion paper ${ }^{2}$.

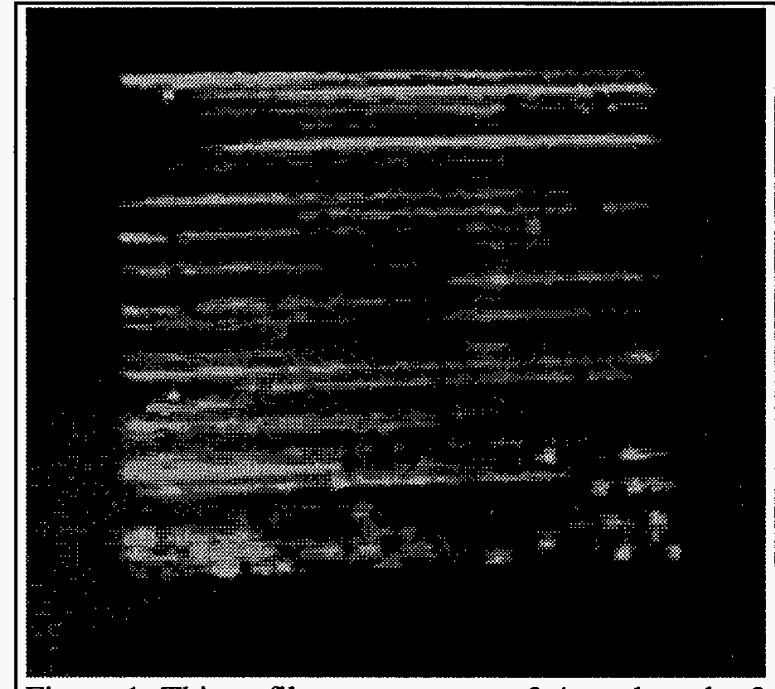

Figure 1 . Thirty filaments across a $3.4 \mathrm{~cm}$ long by 3 $\mathrm{cm}$ wide GaAs PCSS. The filaments are triggered and viewed through a layer of $1 \mathrm{~mm}$ dia. glass rods above the GaAs wafer. In this test the switch was charged to $60 \mathrm{kV}$ and conducted $380 \mathrm{~A}$.

Ground penetrating radar detects underground objects with wide-band RF impulse radiation. Switching goals are $100 \mathrm{kV}, 1-2 \mathrm{kA}$ for $3 \mathrm{~ns}$ at $1-1000 \mathrm{~Hz}$. The benefits of PCSS are high peak power, fast rise time, compact size, and small jitter. Issues are lifetime and post-pulse noise. We have demonstrated the ability of GaAs switches to create voltage pulses suitable for driving ultra wideband (UWB) transmitters ${ }^{4,5}$. In that test we charged a nominally $3.0 \mathrm{~ns}$ long, 
$50 \Omega$, parallel plate transmission line to voltages of about $100 \mathrm{kV}$. This line was discharged with either one or two switches into a $50 \Omega$ load or a TEM horn antenna. The radiated pulse and reflections from a different targets have been measured. Ground penetrating tests will commence in October, 1997.

Firing sets are used to initiate conventional and nuclear explosives. They consist of a power supply, high voltage capacitor, high voltage switch, and a resistive detonator. Switching goals are $1-5 \mathrm{kA}$ at $1-5 \mathrm{kV}$ for $50 \mathrm{~ns}$, with 100 pulse lifetime for testing. The system requirements on the high voltage switch demand precise timing, small volume, high voltage, high current, and very low inductance to produce fast current rise times with a sub-Ohm load. PCSS are being developed to replace present switch technology because of their extremely fast switching and small physical volume. We have tested a system ${ }^{6}$ that consists of a $120 \mathrm{nF}$ ceramic capacitor which is discharged through the switch into a $0.25 \Omega$ load. The capacitor is either DC or pulse charged to $3 \mathrm{kV}$. When triggered, the switch current has a rise time of $<30 \mathrm{~ns}$ with a peak current of $3 \mathrm{kA}$. Issues are the on-state voltage loss and device lifetime.

Laser diode drivers are used to generate high peak power optical pulses by injecting short, high current pulses into semiconductor laser junctions. If the peak current is high enough and the pulse rise time is sufficiently short, the semiconductor lasers will achieve a substantial population inversion before lasing initiates. This sudden increase in laser gain, called gain switching, produces sub-nanosecond optical pulses with optical peak powers that depend on the size and type of semiconductor laser diodes. These optical pulses are used in research laboratories and for military and industrial applications such as active optical sensors for optical range sensing, 3D laser radar, and high speed range-gated imaging. We have demonstrated $100 \mathrm{~nJ}$ in $75 \mathrm{ps}(1.3$ $\mathrm{kW}$ ) from individual, single heterojunction, edge-emitting lasers ${ }^{3,7}$. We have used PCSS to drive arrays of 20,100, and 600 lasers with total pulse energies of 2,12 , and $48 \mu \mathrm{J}$, respectively. PCSS switching goals are 200-1000 A at 1-15 kV depending of the size and electrical configuration of the laser diode arrays (LDA). The effective impedance of each individual laser is less than $1 \Omega$, so parallel configurations, which are the standard monolithic configuration, can be extremely low impedance. In the low impedance $(R)$ regime, the rise time of any driving. circuit is usually dominated by the circuit inductance $(\mathrm{L})$ and is given by $\mathrm{L} / \mathrm{R}$. The benefits of using PCSS for this application are its low inductance, rise time, peak currents, repetition rate. Primary issues for this applications are device lifetime and jitter.

Electro-optic (Pockels cell) drivers are used for electrically-driven optical switching. Such devices are used in lasers for Q-switches, cavity dumpers, pulse selectors, and regenerative amplifiers. Switching goals are $1-8 \mathrm{kV}$ in $50 \Omega$ systems, sub-nanosecond rise times and in some cases $\mathrm{kHz}$ or even $\mathrm{MHz}$ repetition rates. The benefits of PCSS are rise time, optical activation, compact size, and low cost. Issues are switch lifetime and jitter for precise synchronization with other systems. We have used PCSS to deliver up to $5 \mathrm{kV}$ to Pockels cells and demonstrated optical rise times of less than $600 \mathrm{ps}^{6}$.

High voltage triggers for gas discharge switches. The switching goals are $50 \mathrm{kV}, 50 \Omega$, and high repetition rates. Benefits of using PCSS are optical activation, compact size and cost. Issues are jitter and device lifetime. We have demonstrated 80 ps and 350 ps rise time with PCSS, and are presently assembling a system to trigger high voltage gas discharge switches with a PCSS. 


\section{PCSS Triggering Experiments}

A vertical cavity surface emitting laser (VCSEL) has been used to trigger a $1 \mathrm{~mm}$ long GaAs PCSS. As shown in Figure 2 below, this VCSEL emits $2.15 \mathrm{~nJ}$ of energy. The peak power of $80 \mathrm{~mW}$ may be underestimated due to the limited bandwidth of the photodetector and oscilloscope used to obtain the measurement. The laser has a "hollow" square emitting aperture of $75 \mu \mathrm{m}$ width, with a lasing wavelength of $850 \mathrm{~nm}$, and is optimized for high output efficiency. A high numerical aperture, aspherical lens was used to image the VCSEL output onto the surface of the switch with nominally unity magnification. The switch was biased at $3.5 \mathrm{kV}$, resulting in a current pulse of $43 \mathrm{~A}$ upon triggering. The integration of high-gain PCSS with trigger lasers has important implications for many applications in terms of manufacturability, reliability, cost, and packaging. VCSELs may play an important role in the implementation of such a device due to their advantages of surface normal emission and ease of array fabrication and on-wafer testing. VCSEL arrays may also eventually be used to initiate multiple filaments. An outstanding issue for VCSEL triggering of PCSS devices has been that of the optical pulse energy required to cause the switch triggering. Previously, the minimum energy that had been observed to achieve switch firing was $13.6 \mathrm{~nJ}$ in a $40 \mu \mathrm{m}$ diameter spot of illumination, at a wavelength of $532 \mathrm{~nm}$.

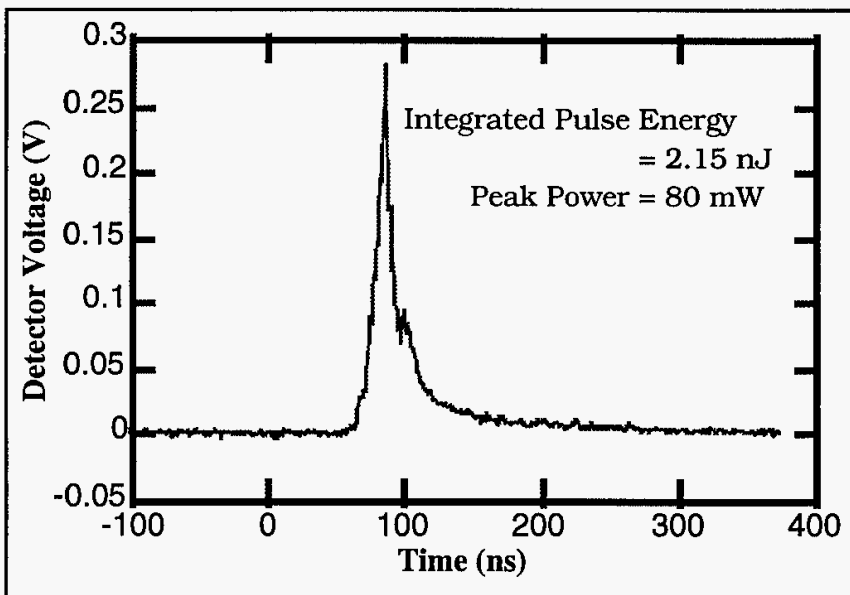

Figure 2. The optical output from a VCSEL which was used to trigger a $1 \mathrm{~mm}$ long GaAs PCSS.

The energy to required to trigger a high gain GaAs PCSS is minimized by focusing it to a point near one of the contacts, where the initial field is apparently highest. However, to initiate multiple filaments on a single wafer, additional optical energy is expended with line-triggering to precisely control the location of the filament. Multiple filaments reduce the switch inductance and increase the total current carrying capacity of the PCSS, as switch longevity is limited by contact damage which depends on the current per filament ${ }^{2}$. PCSS line-triggering experiments have produced thirty filaments on a single GaAs wafer (Figure 1). The trigger pulses were transported to the switch in thirty-two $200 \mu \mathrm{m}$ diameter fibers $(0.21 \mathrm{NA})$. The average optical energy per fiber was $15 \mu \mathrm{J}$ of $532 \mathrm{~nm}$ light. The light from the fibers was focused into lines with a layer of $1 \mathrm{~mm}$ diameter glass rods placed directly on the surface of the PCSS. The fibers were grouped into a line of 8 bundles separated by $2 \mathrm{~mm}$ and $18 \mathrm{~cm}$ above the glass rods. The switch and glass rods were submerged in Fluorinert to inhibit electrical breakdown near the surface of the switch. Figure 1 shows a variation in the intensity along the individual filament lines. Part of this variation is caused by viewing the image of the filaments through the layer of glass rods. If the filaments do not form directly below the rods with respect to the camera, their emitted light is not directed toward the camera. Another contribution to the variation is that in some regions the filaments are formed deeper in the GaAs and more of the emission is re-adsorbed by the GaAs. 
The jitter, or variation in the delay between the optical trigger pulse and the onset of switching, was measured in a $1 \mathrm{~mm}$ long GaAs PCSS charged to $4 \mathrm{kV}$. It was triggered with a single laser diode modified for fast rise time with approximately $1 / 2 \mu \mathrm{J}$ of $880 \mathrm{~nm}$ light. A 4 $\mathrm{GHz}$ transient digitizer and high speed PCSS circuit was used to make this measurement. The trigger pulses were delivered through a $300 \mu \mathrm{m}$ diameter fiber optic placed near the surface of the switch. Ninety-six successive current waveforms were recorded. The root-mean-square variation was 80 ps and the first-to-last spread was 390 ps. There has been some indication that line triggering may reduce the jitter further by eliminating some of the statistical variation in filament formation. We plan to repeat this experiment with line triggering.

\section{PCSS Imaging Experiments}

Time-resolved images of current filaments (Figure 3) were obtained using a Schlieren imaging technique ${ }^{8}$. The technique images small changes in the refractive index of a the semiconductor substrate due to current density and temperature. In this experiment, one LDA was used to trigger the PCSS with $850-880 \mathrm{~nm}$ radiation which is strongly absorbed in GaAs. Another LDA is used to illuminate switch with $904 \mathrm{~nm}$ which is only weakly absorbed in GaAs. Images of the refractive index variations during the second pulse are captured with a CCD camera. Time resolution is obtained by using short optical pulses varying the delay between them. In Figure 3, the trigger pulse and the imaging pulse were $13 \mathrm{~ns}$ wide. Our original results were obtained with 300 ps pulses from an ND:YAG laser. The coherence length of that laser produced speckle patterns which seriously degraded the images. Using an LDA eliminated speckle. In our next experiments, we plan to improve time resolution and magnification.

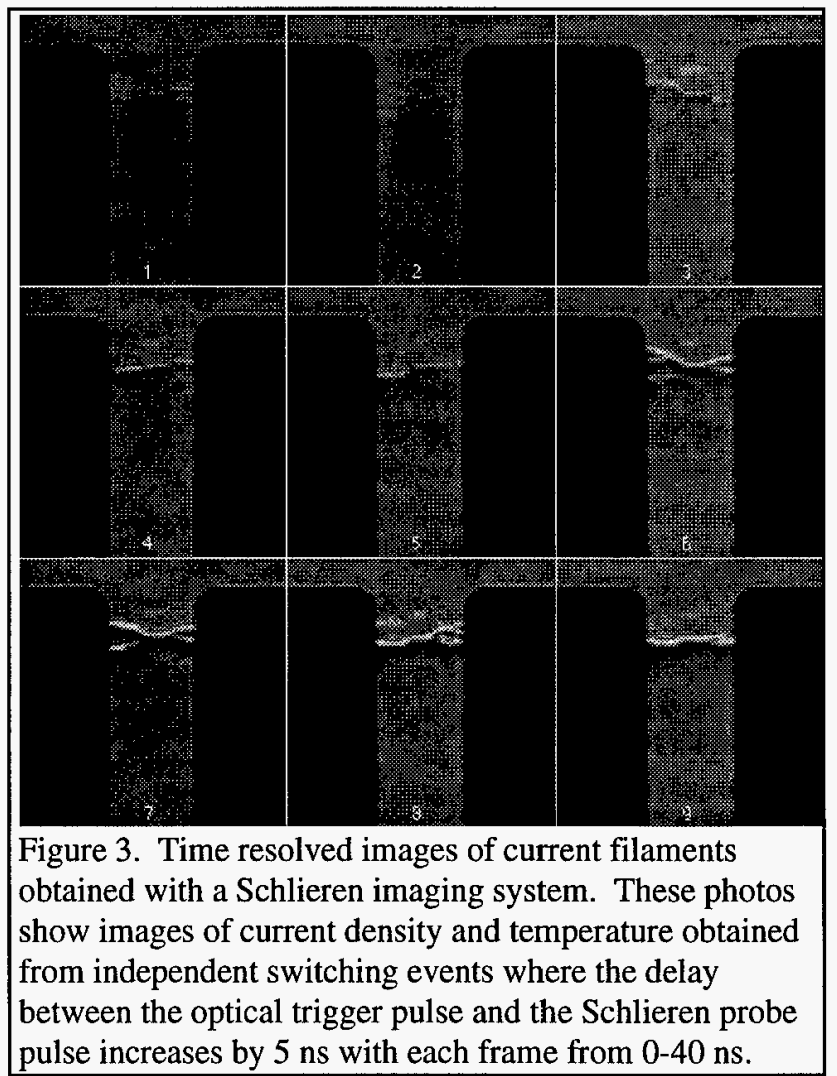

Figure 3. Time resolved images of current filaments obtained with a Schlieren imaging system. These photos show images of current density and temperature obtained from independent switching events where the delay pulse increases by $5 \mathrm{~ns}$ with each frame from $0-40 \mathrm{~ns}$.

\section{High Gain PCSS Modeling}

Theory predicts that semi-insulating (SI) GaAs can exhibit bistable switching. Normally, SI GaAs has a highly resistive OFF state ${ }^{9}$. Under the right conditions it can be optically triggered into a highly conductive ON state in which the current flows in filaments. The mechanism for this process is collective impact ionization which occurs at high carrier density when carriercarrier scattering is faster than carrier-phonon scattering. In this limit, an electric field $(5 \mathrm{kV} / \mathrm{cm})$ is able to sustain a stable filament by impact ionization. Figure 4 shows a series of plots of electron and hole carrier density as a function of time in a $100 \mu \mathrm{m}$ switch. The contacts have 
dopant concentrations of $10^{18} \mathrm{~cm}^{-3}$ and thicknesses of $10 \mu \mathrm{m}$. An initial $1 \mathrm{ps}$ excitation pulse of Gaussian profile generates a neutral plasma of electrons and holes. The logarithmic time scale has 5 steps per decade. A forward bias $(100 \mathrm{~V})$ causes electrons to begin impact ionization at both the n-contact (left) and the right-hand-side of the plasma. For each electron, a hole is generated and it flows to the left. At the end of the $1 \mathrm{~ns}$ calculation, the filament carrier density is nearly uniform except near the contacts. At the n-contact, the holes "pile-up" which leads to increased electron injection. The net result is a reduced electric field and carrier temperature in the contact. A similar phenomenon occurs in the p-contact due to "pile-up"

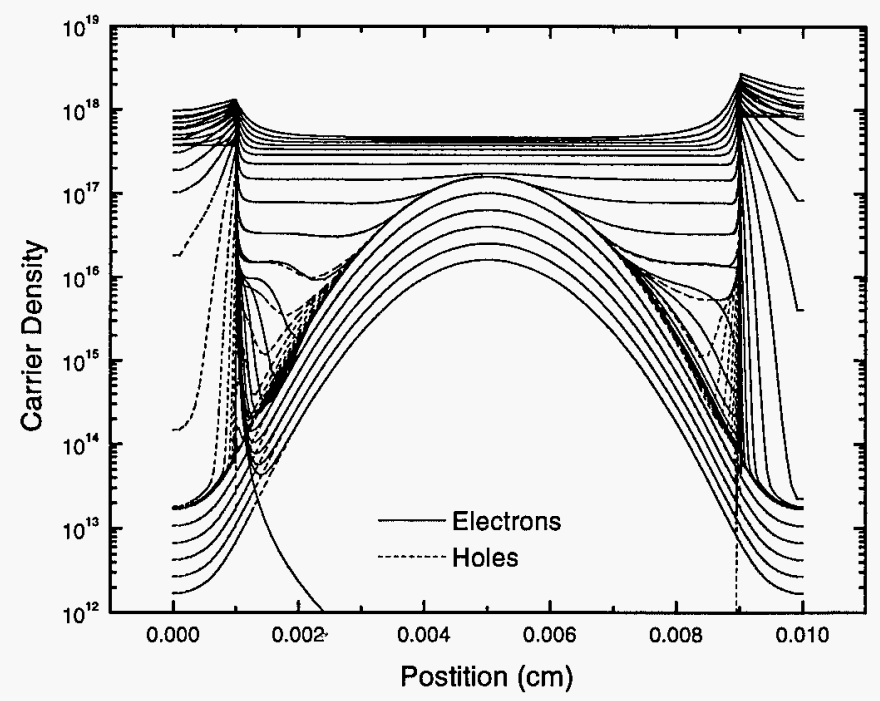

Figure 4. This 1-d transport calculation is capable of showing the effect excitation conditions, circuit conditions, doping, and switch geometry on the switch voltage, current, rise time, and temperature.

of the electrons. This model is now being used to improve switch lifetime and predict the performance of the switches.

\section{References}

1. G. M. Loubriel, F. J. Zutavern, A. G. Baca, H. P. Hjalmarson, T. A. Plut, W. D. Helgeson, M. W. O'Malley, M. H. Ruebush, and D. J. Brown, "Photoconductive Semiconductor Switches," IEEE Transactions on Plasma Science, 25, 1997, pp. 124-130.

2. G. M. Loubriel, F. J Zutavern, A. Mar, M. W. O’Malley, W. D. Helgeson, D. J. Brown, H. P. Hjalmarson, and A. B. Baca, "Longevity of Optically Activated, High Gain Ga As Photoconductive Semiconductor Switches," to be published in Proc. of $11^{\text {th }}$ IEEE Pulsed Power Conference, Baltimore, MD, 1997.

3. F. J. Zutavern, G. M. Loubriel, W. D. Helgeson, M. W. O'Malley, M. H. Ruebush, H. P. Hjalmarson, and A. G. Baca, "Optically-Activated GaAs Switches for Compact Accelerators", Proc. 22nd Power Modulator Symposium, Boca Raton, FL, June 24-27, 1996, pp. 31-34.

4. G. M. Loubriel, F. J. Zutavern, W. D. Helgeson, D. J. Brown, and M. W. O'Malley, "High Gain GaAs Switches for Ground Penetrating Radar", Proc. 22nd Power Modulator Symposium (IEEE, NY, 1996), Boca Raton, FL, June 24-27, 1996, pp. 165-168.

5. G. M. Loubriel, M. T. Buttram, J. F. Aurand, and F. J. Zutavern, "Ground Penetrating Radar Enabled by High Gain gas Photoconductive Semiconductor Switches," in Ultra-Wideband, Short Pulse Electromagnetics 3, A. Stone, C. Baum, and L. Carin, eds., Plenum Press, NY, 1996, pp. 17- 24.

6. G. M. Loubriel, F. J. Zutavern, A. G. Baca, H. P. Hjalmarson, W. D. Helgeson, and M. W. O'Malley, "Photoconductive Semiconductor Switches for Firing Sets and Electro-Optic Modulators," Proceedings of 10th IEEE Pulsed Power Conference, Albuquerque, NM, July 10- 13, 1995, pp. 354- 359.

7. Fred J Zutavern, and Wesley D. Helgeson, Guillermo M. Loubriel, George J. Yates, Robert A. Gallegos, and Thomas McDonald, "A Compact, Short-pulse Laser for Near-field, Range-gated Imaging," in Proc. of SPIE 22nd International Congress on High-Speed Photography and Photonics, Santa Fe, NM, 1996.

8. R. Aaron Falk, F. J. Zutavern, M.W. O'Malley, “Carrier Density and Thermal Images of Transient Filaments in GaAs Photoconductive Switches, Proc. SPIE Conf. On DiagnosticTechniques for Semiconductor Materials and Devices, Montreal, 1997.

9. H. P. Hjalmarson, F. J. Zutavern, G. M. Loubriel, L. A. Romero, A. G. Baca, K. Khachaturyan, and D. R. Wake, "An Impact Ionization Model For Optically-Triggered Current Filaments in GaAs", SAND93-3972, SNL, 1996. 
M98000185

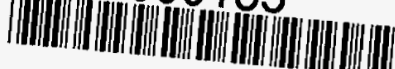

Report Number (14) SAND-96-3015C

Publ. Date (11) $\frac{199709}{\text { Sponsor Code (18) DOELMA, XF }}$

199709

UC Category (19) UC-900, DOE/ER 